\title{
Effects of ageing on the eyes
}

AKADÉMIAI KIADÓ

Developments in Health Sciences

$4(2021) 1,21-25$

DOI:

(c) 2021 The Author(s)

\section{REVIEW ARTICLE}

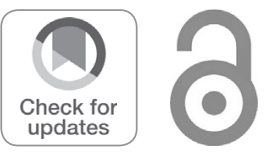

10.1556/2066.2021.00047

\section{KOVÁCS ${ }^{1,2 *}$ []}

${ }^{1}$ Department of Clinical Ophthalmology, Faculty of Health Sciences, Semmelweis University, Budapest, Hungary

${ }^{2}$ Department of Ophthalmology, Faculty of Medicine, Semmelweis University, Budapest, Hungary

Received: July 12, 2021 • Revised manuscript received: October 15, 2021 • Accepted: November 2, 2021

Published online: January 7, 2022

\section{ABSTRACT}

With ageing, significant changes occur in all structures of the eye, resulting in a variety of morphological and functional effects. This review summarises parameters that are within the normal ageing process in order to distinguish them from true disease processes. Understanding the ageing changes of the eye will help to understand some of the visual problems experienced by the ageing population.

\section{KEYWORDS}

eye diseases, ageing, eyelid abnormality, vitreous floaters

\section{INTRODUCTION}

The eye is a highly specialised sensory organ. It collects light and focuses onto the retina at the back of the eye, where light is converted into nerve impulses. The optic nerve transmits these electrical signals to the brain, which forms an image so thereby providing sight. As we age, the functional capabilities of the eye decline, and so does the central visual system. Common age-related ocular problems include lid malpositions, presbyopia, glaucoma, dry eyes, cataracts, vitreous floaters, and age-related macular degeneration. Industrialised nations are ageing societies with a rapidly growing proportion of people over age 65, and in many countries, this is the fastest-growing segment of society. Everyone hopes to age without visual impairment, but that is rarely possible. Most of the major eye diseases are age-related, and the prevalence of sight-threatening diseases increases dramatically after the age of 75 . In the United States, 4.2 million people are blind or have a significant visual impairment, which number shows an increase of nearly $30 \%$ over the past decade. These numbers demonstrate their impact on individuals and on our society, both in terms of healthcare costs and of decreased quality of life.

Many theories have been proposed to explain the ageing process, but none of them seems entirely satisfactory. Recent biological theories of ageing in humans fall into two main categories: programmed theories and damage or failure theories [1]. The programmed theories imply that ageing follows a biological timetable, perhaps a continuation of that which regulates growth and development in childhood. This regulation would depend on changes in gene expression that affect the systems responsible for maintenance, repair, and defence responses. The damage or failure theories emphasise environmental attacks on living organisms that induce cumulative damage at various levels as the cause of ageing.
*Corresponding author. Department of Clinical Ophthalmology, Faculty of Health Sciences, Semmelweis University, Vas u. 17, H-1088, Budapest, Hungary.

E-mail: kovacs.illes@se-etk.hu

\section{The programmed theory has three subcategories}

1) Programmed Longevity: this hypothesis explains ageing as the result of sequential on and off switching of certain genes, with senescence defined as the point at which age-related deficits manifest themselves [2]; 2) Endocrine Theory: biological clocks act through hormones to control the pace of ageing. Recent studies confirm that ageing is hormonally regulated and that the evolutionarily conserved insulin/IGF-1 pathway plays a key role in the hormonal 
regulation of ageing [3]; 3) Immunological Theory: the immune system is programmed to decline over time, leading to increased susceptibility to infectious disease and consequent ageing and death. It is well documented that the effectiveness of the immune system peaks at puberty and then gradually declines with age. A dysregulated immune response, at least indirectly, has been linked to cardiovascular disease, inflammation, Alzheimer's disease, and cancer $[4,5]$.

The damage or defect theory includes 1) wear and tear theory: cells and tissues have vital parts that wear out over time, leading to ageing. According to the theory of ageing, introduced by Dr August Weismann in 1882, parts of the body eventually wear out from repeated use, with subsequent damage to the tissues and then to the body. 2) Rate of living theory: The basic idea of this hypothesis is that the higher the rate of basic oxygen turnover, the shorter the life span of an organism [6]; 3) Cross-linking theory: According to this theory, an accumulation of cross-linked proteins damages cells and tissues, slowing down bodily processes, leading to ageing [7]; 4) Free radicals theory: This theory states that superoxide and other free radicals damage the macromolecular components of the cell, leading to an accumulation of damage that disables cells and eventually organs. Macromolecules such as nucleic acids, lipids, sugars, and proteins are especially vulnerable to free radical attack. The human body has natural antioxidants in the form of enzymes that prevent the dangerous accumulation of these free radicals, without which the cellular death rate would greatly increase and life expectancy would significantly decrease [8]. 5) Somatic DNA damage theory: DNA damage occurs constantly in the cells of living organisms. While most of this damage is repaired, some accumulate as genetic mutations with age, causing cells to deteriorate. In particular, damage to mitochondrial DNA can lead to mitochondrial dysfunction.

\section{Ageing changes of the eyelids}

The involutional changes of the eyelids reflect similar changes that occur throughout the face. Gradual tissue atrophy results in redundant skin folds and wrinkles. Loss of adnexal structural support of the tarsus, canthal tendons, and orbicularis muscle results in orbital fat prolapse, eyelid malposition, and blepharochalasis. Eyelid malpositions are more common on the lower eyelid. Age-related reduction of orbital fat causes the eyes to sink into the orbits, exacerbating eyelid laxity. As laxity progresses, punctual eversion and later eversion of the lid margin from the bulb (ectropion) may occur, resulting in a watery eye (Fig. 1). If the pretarsal orbicularis muscle is comparatively strong, inversion of the eyelid (entropion) may occur instead, resulting in rubbing of the eyelashes against the cornea and subsequent ocular surface discomfort (Fig. 2). In the upper eyelids, failure or weakening of the levator muscle may lead to involutional ptosis [1,9]. Excess upper eyelid skin along with the advancement of the preaponeurotic fat pads results in dermatochalasis. Watery eye in the elderly is mainly

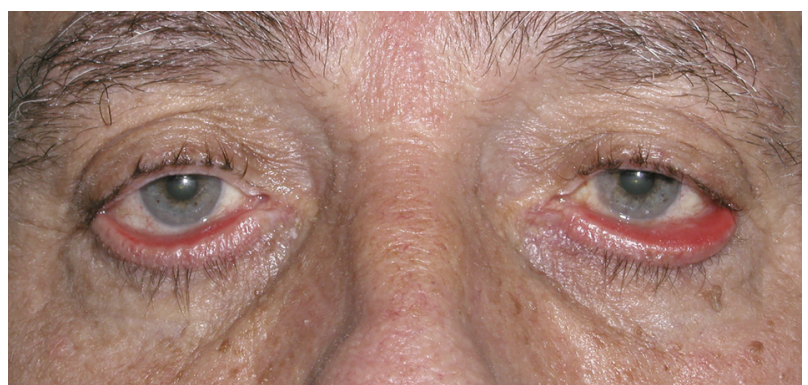

Fig. 1. Ectropium of the lower eyelids

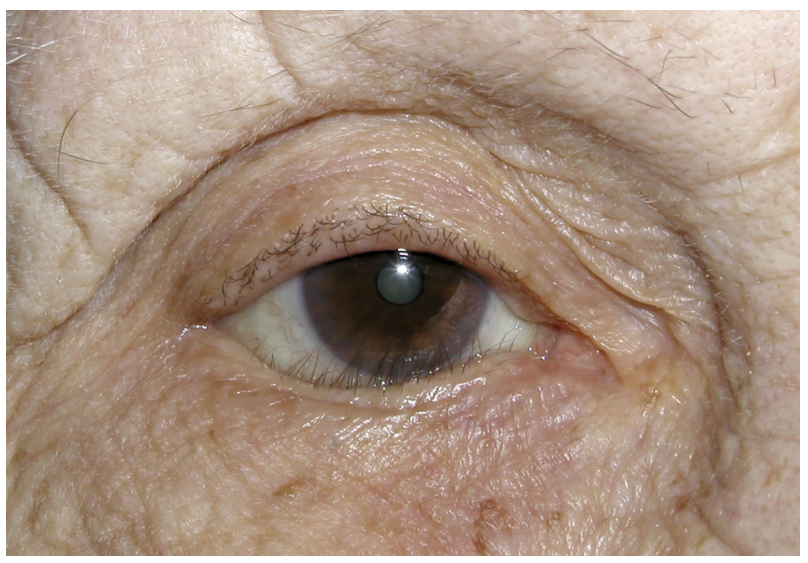

Fig. 2. Entropium of the lower eyelid and senile miosis

caused by eyelid malposition such as ectropion [10] as well as by impaired tear secretion and often leads to recurrent bacterial conjunctivitis (Fig. 3).

\section{Ageing changes of tear secretion}

In the elderly, usually, there is a decrease in the quantity and/or quality of tears produced by the lacrimal glands, causing symptoms of dry eye. The evaporative form of dry eye and Meibomian gland dysfunction (MGD) show a strong positive correlation with ageing. Several previous studies have observed a significant association between abnormalities of the lid margin or Meibomian glands and

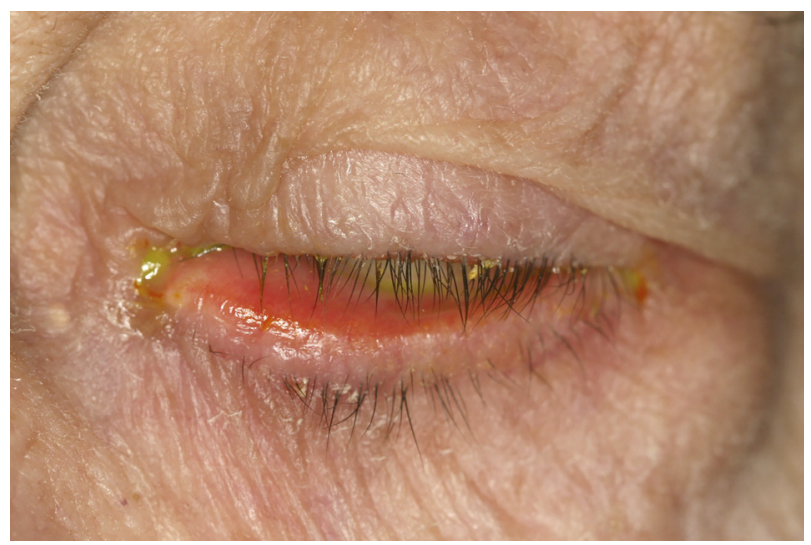

Fig. 3. Bacterial conjunctivitis due to lower lid ectropium 
ageing [11]. Ageing is associated with a significant increase in lower eyelid oedema, telangiectasia, keratinisation, Meibomian gland orifice metaplasia, and thick, opaque meibomian secretion [12]. The number of active Meibomian glands also decreases by half between the ages of 20 and 80 years [13], and glandular dropout is often seen in the elderly [14]. These structural and functional changes lead to the development of dry eye symptoms, which are usually treated with artificial tears to improve the stability of the tear film on the ocular surface.

\section{Corneal changes}

Age-related changes in corneal curvature cause a change in refraction, primarily in the refractive power and the meridian of astigmatism. Therefore regular refraction cheques are recommended for the elderly to ensure optimal vision. Other corneal changes include a decrease in corneal sensitivity, which can lead to the development of dry eye symptoms. Arcus senilis is the most noticeable and common ageing corneal change. These asymptomatic bilateral yellowwhite deposits of cholesterol esters usually begin inferiorly and then superiorly form an annular opacity on the peripheral corneal stroma, separated from the limbus by a narrow band of clear cornea [15]. Ageing can affect the innermost layer of the cornea, and the endothelium layer as well, which cannot regenerate, so endothelial cell density decreases with age. The remaining endothelial cells enlarge to cover the loss of the endothelial cells with age. Because endothelial cells maintain corneal clarity, a further decrease in endothelial cell number beyond a certain level can lead to corneal oedema and thickening and subsequent decrease in visual acuity. The deposition of uveal pigment on the corneal endothelium with age (Kruckenberg spindle) usually has no effect on visual acuity [1].

\section{Trabecular meshwork and uveal changes}

With ageing, there is increased pigmentation of the trabecular meshwork resulting in increased resistance to aqueous humour outflow. Significant age-related resistance can lead to an increase in intraocular pressure and the development of glaucoma. With ageing, the pupil tends to become smaller and less reactive to light (senile miosis) and also more difficult to dilate pharmacologically (Fig. 2). The loss of pigment in the iris can cause iris transillumination on slit lamp examination, particularly at the pupil margin. The shape and tone of the ciliary body change with age, and this, together with changes in the decreasing elasticity of the crystalline lens leads to a decreased amplitude of accommodation, resulting in presbyopia. The reduction in near vision caused by presbyopia can usually be compensated for by holding objects at a greater distance or by using near correction glasses (bifocals, reading glasses) [1].

\section{Crystalline lens changes}

With age, the crystalline lens selectively absorbs more blue light as yellow pigments accumulate in the lens. This decrease in the transmittance of blue light is part of the cataractogenic process and causes relative "blue blindness", exemplified by the increase in brown colour in paintings by famous painters as they age. One of the most important consequences of ageing is the hardening of the lens caused by various biochemical changes. Symptoms of presbyopia appear in patients many years before cataract formation. Presbyopia, as mentioned earlier, is due to the change in capsular elasticity and lens hardening as a result of agerelated changes in lens protein solubility [16]. Starting in their early to mid-forties, many adults have trouble seeing clearly at near, especially when reading or working on a computer. This is among the most common eye problems in adults which develop between the ages of 40 and 60 . This normal change in the eyes' ability to focus, called presbyopia, will continue to progress over time. Initially, subjects may need to hold reading materials farther away to see them clearly. Writing in the newspaper or on a restaurant menu may appear blurry, especially in low light. With ageing, presbyopia progress and subjects need a regular change in reading glasses. Around age 60 , these changes in near vision usually stop and prescription changes occur less frequently. Presbyopia cannot be prevented or cured, but reading glasses provide subjects with a clear and comfortable near vision that meets all of their lifestyle needs. Cataract surgery and intraocular lens implantation are performed in patients whose quality of life and performance of routine daily activities are negatively affected by the presence of significant lens opacities such as cataract (Fig. 4).

\section{Vitreous ageing}

The vitreous undergoes an irreversible ageing process characterised by changes in the collagen fibrils and hyaluronic acid components, causing harmless floaters perceived by the elderly. Clinically, ageing results in a compaction of the vitreous gel, enlargement of the fibrillar structure of the vitreous, increased mobility of the fibrillar structures, and the formation of optically empty spaces called lacunae (Fig. 5). As vitreous liquefaction progresses, the resulting

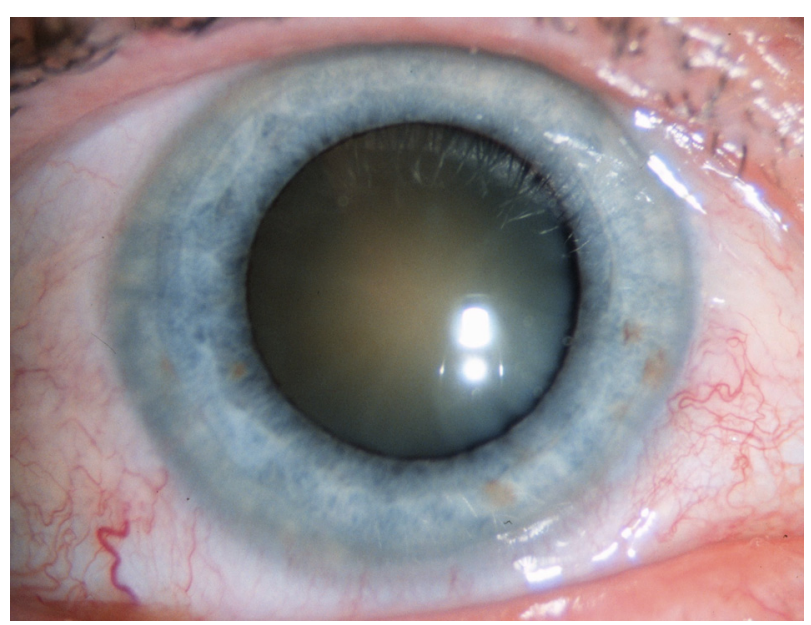

Fig. 4. Age-related nuclear sclerosis in the crystalline lens 


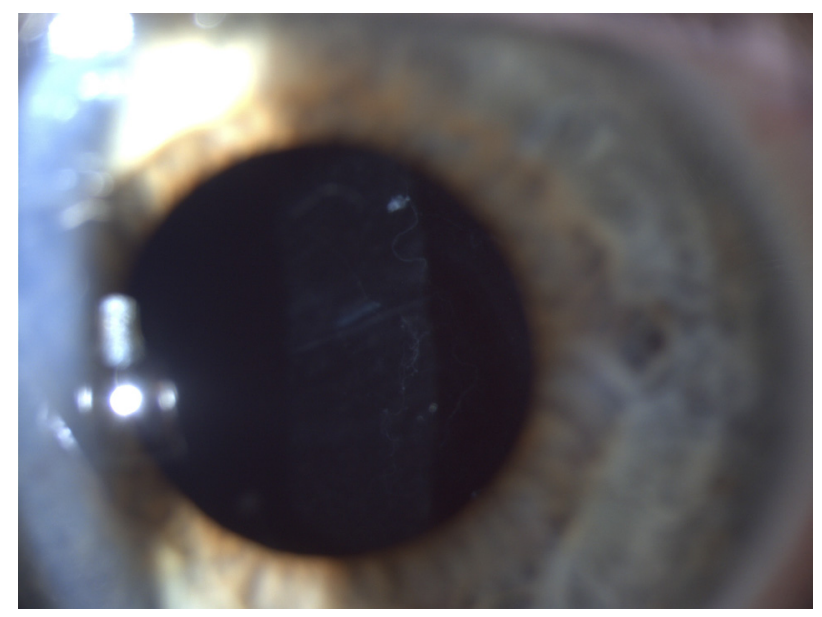

Fig. 5. Vitreous floaters posterior to an intraocular lens

lacunae grow together and form larger cavities, eventually leading to shrinkage of the vitreous from the retina, named posterior vitreous detachment (PVD) [1]. PVD is harmless and patients might notice floaters in front of the eye that moves in the direction of gaze. Occasionally, severe vitreoretinal adhesion in the peripheral retina during acute PVD may result in a retinal tear that requires laser treatment to prevent the progression to retinal detachment. Therefore, patients with sudden flashes of light (caused by traction on the retina during PVD) and floaters (caused by either vitreous debris or pigment dispersion from the ruptured retina) must be referred to an ophthalmologist to rule out retinal tears associated with PVD. The appearance of a curtain-like shadow in the visual field may indicate a retinal detachment requiring prompt surgical intervention.

\section{Ageing in the retina}

Visual quality deteriorates with age, and virtually every measure of visual function shows declining performance with age, including decreasing visual acuity, decreasing visual field sensitivity, decreased contrast sensitivity, and increased dark adaptation threshold. Declining visual function is a combination of mainly age-related changes in the neural elements of the retina and changes in the ocular media [1]. The retinal pigment epithelium (RPE), which is essential for the integrity of the photoreceptors, shows decreased melanin content, increased lipofuscin content, decreased cytoplasmic volume, and areas of atrophy with age. It has already been shown that in normal subjects the retinal microcirculation decreases with age as well [17]. Grunwald et al. also showed a decrease in choroidal blood flow parameters with an increase in the severity of agerelated macular degeneration, suggesting a direct link between them [18].

\section{Age-related macular degeneration}

Age-related macular degeneration (AMD) is a slowly progressing neurodegenerative disease of the central part of the retina (macula lutea) affecting the photoreceptors, retinal pigment epithelium (RPE), and also the underlying choriocapillaris and connective tissue (Bruch's membrane) [19]. In the literature, there has been much debate over which factor is the primary cause of the disease. There is accumulating evidence that a dynamic interaction may exist: a cross-talk that occurs physiologically between the photoreceptor/ pigment epithelial metabolism and the blood perfusion of the choriocapillaris. In normal conditions, it adapts to the continuously changing functional demands, but it is affected by ageing and certain risk factors [20]. Due to these agerelated changes aggravated by risk factors (smoking, UV exposure), the regeneration of photoreceptors becomes incomplete and metabolic by-products are deposited in the Bruch's membrane. These substances alter the permeability of Bruch's membrane making the metabolism of photoreceptors and RPE more inefficient [21]. The release of proinflammatory substances from the RPE causes inflammatory reactions which clinically and pathologically manifest as AMD [22], while in other cases excessive release of reactive oxygen species (ROS) dominates the pathophysiology [23].

\section{Functional changes}

Vision becomes impaired in various ways as we age. Presbyopia is a conspicuous feature of the physiological insufficiency of accommodation associated with ageing that results in progressively worsening ability to focus clearly on close objects. Older people with early nuclear sclerosis may complain of glare because the light is scattered in the crystalline lens. These symptoms are more pronounced in the dark, and subjects affected require more ambient lighting than a younger person. Decreased contrast sensitivity also decreases an older person's ability to perceive depth and makes it difficult to perceive steps or street curves. Therefore, elderly patients who can drive well during the day may have difficulty driving at night. A reduction in the visual field is normal with age and a reduction in the number of cones on the fovea leads to a reduction in colour vision. In the elderly, blue light is attenuated by the ageing lens, causing a yellowish appearance of objects.

\section{CONCLUSIONS}

The effects of age-related changes in the eye have a significant impact on the quality of life of the elderly. When considering the effects of ageing on vision, it is important to distinguish between reduced visual function in the normal healthy elderly person and the elderly patient with specific age-related diseases [24]. In the future, interventions may become possible to prevent or avoid many age-related visual impairments and thus improve the quality of life of this growing population.

Authors' contribution: NA.

Ethical approval: NA. 
Conflicts of interest: The authors declare no conflict of interest and no financial support was received for this study.

Acknowledgements: NA.

\section{REFERENCES}

1. Salvi SM, Akhtar S, Currie Z. Ageing changes in the eye. Postgrad Med J 2006;82:581-7. https://doi.org/10.1136/pgmj.2005.040857.

2. Davidovic M, Sevo G, Svorcan P, Milosevic DP, Despotovic N, Erceg P. Old age as a privilege of the "selfish ones". Aging Dis 2010; $1: 139-46$.

3. van Heemst D. Insulin, IGF-1 and longevity. Aging Dis 2010;1: $147-57$.

4. Rozemuller AJ, van Gool WA, Eikelenboom P. The neuroinflammatory response in plaques and amyloid angiopathy in Alzheimer's disease: therapeutic implications. Curr Drug Targets CNS Neurol Disord 2005;4:223-33. https://doi.org/10.2174/ 1568007054038229.

5. Jin K. Modern biological theories of aging. Aging Dis 2010;1:72-4.

6. Brys K, Vanfleteren JR, Braeckman BP. Testing the rate-of-living/ oxidative damage theory of aging in the nematode model Caenorhabditis elegans. Exp Gerontol 2007;42:845-51. https://doi.org/10. 1016/j.exger.2007.02.004.

7. Bjorksten J. The crosslinkage theory of aging. J Am Geriatr Soc 1968;16:408-27. https://doi.org/10.1111/j.1532-5415.

8. Afanas'ev I. Signaling and damaging functions of free radicals in aging-free radical theory, hormesis, and TOR. Aging Dis 2010;1:75-88.

9. Van den Bosch WA, Leenders I, Mulder P. Topographic anatomy of the eyelids, and the effects of sex and age. Br J Ophthalmol 1999; 83:347-52. https://doi.org/10.1136/bjo.83.3.347.

10. Van Haeringen NJ. Aging and the lacrimal system. Br J Ophthalmol 1997;81:824-6. https://doi.org/10.1136/bjo.81.10.824.

11. Den S, Shimizu K, Ikeda T, Tsubota K, Shimmura S, Shimazaki J. Association between meibomian gland changes and aging, sex, or tear function. Cornea 2006;25:651-5. https://doi.org/10.1097/01.ico. 0000227889.11500.6f.

12. Sullivan BD, Evans JE, Dana MR, Sullivan DA. Influence of aging on the polar and neutral lipid profiles in human meibomian gland secretions. Arch Ophthalmol 2006;124:1286-92. https://doi.org/10. 1001/archopht.124.9.1286.
13. Norn M. Expressibility of meibomian secretion. Relation to age, lipid precorneal film, scales, foam, hair and pigmentation. Acta Ophthalmol (Copenh) 1987;65:137-42. https://doi.org/10.1111/j. 1755-3768.1987.tb06991.x.

14. Arita R, Itoh K, Inoue K, Amano S. Noncontact infrared meibography to document age-related changes of the meibomian glands in a normal population. Ophthalmology 2008;115:911-5. https://doi. org/10.1016/j.ophtha.2007.06.031.

15. Faragher RG, Mulholland B, Tuft SJ, Sandeman S, Khaw PT. Aging and the cornea. Br J Ophthalmol 1997;81:814-7. https://doi.org/10. 1136/bjo.81.10.814.

16. Duncan G, Wormstone IM, Davies PD. The aging human lens: structure, growth, and physiological behaviour. Br J Ophthalmol 1997;81:818-23. https://doi.org/10.1136/bjo.81.10.818.

17. Grunwald JE, Piltz J, Patel N, Bose S, Riva CE. Effect of aging on retinal macular microcirculation: a blue field simulation study. Invest Ophthalmol Vis Sci 1993;34:3609-13.

18. Grunwald JE, Metelitsina TI, Dupont JC, Ying GS, Maguire MG. Reduced foveolar choroidal blood flow in eyes with increasing AMD severity. Invest Ophthalmol Vis Sci 2005;46:1033-8. https:// doi.org/10.1167/iovs.04-1050.

19. Zarbin MA. Current concepts in the pathogenesis of age-related macular degeneration. Arch Ophthalmol 2004;122:598-614. https://doi.org/10.1001/archopht.122.4.598.

20. Feigl B, Brown B, Lovie-Kitchin J, Swann P. Functional loss in early age-related maculopathy: the ischaemia postreceptoral hypothesis. Eye (Lond). 2007;21:689-96. https://doi.org/10.1038/sj. eye.6702389.

21. Roth F, Bindewald A, Holz FG. Keypathophysiologic pathways in age-related macular disease. Graefes Arch Clin Exp Ophthalmol 2004;242:710-6. https://doi.org/10.1007/s00417-0040976-x.

22. Bhutto IA, McLeod DS, Hasegawa T, Kim SY, Merges C, Tong P, Lutty GA. Pigment epithelium-derived factor (PEDF) and vascular endothelial growth factor (VEGF) in aged human choroid and eyes with age-related macular degeneration. Exp Eye Res 2006;82(1): 99-110. https://doi.org/10.1016/j.exer.2005.05.007.

23. Shen JK, Dong A, Hackett SF, Bell WR, Green WR, Campochiaro PA. Oxidative damage in age-related macular degeneration. Histol Histopathol 2007;22:1301-8. https://doi.org/10.14670/HH-22. 1301.

24. Forrester JV. Aging and vision. Br J Ophthalmol 1997;81:809-10. https://doi.org/10.1136/bjo.81.10.809. 